\title{
Dynamical vacuum energy via adjustment mechanism
}

\author{
A.D. Dolgov and F.R. Urban \\ ITEP, Bolshaya Cheremushkinskaya 25, 117218, Moscow, Russia \\ INFN Sezione di Ferrara, via Saragat 1, 44100 Ferrara, Italy \\ Dipartimento di Fisica, Università di Ferrara, via Saragat 1, 44100 Ferrara, Italy
}

\begin{abstract}
A new mechanism of adjustment of vacuum energy down to the observed value from an initially huge one is considered. The mechanism is based on a very strong variation of the gravitational coupling constant in very early universe. The model predicts that the noncompensated remnant of vacuum energy changes very slowly at late stages of the cosmological evolution and is naturally close to the observed one. Asymptotically the effective vacuum energy tends to a negative value, so the cosmological expansion should stop and turn into contraction. There remains the problem of introduction of the usual matter into the model and therefore realising realistic cosmology.
\end{abstract}

\section{INTRODUCTION}

The life-story of vacuum energy is quite dramatic. After it was introduced in 1918 by Einstein [1] under the name cosmological constant or Lambda-term, it did not live long. The Hubble discovery of the cosmological expansion [2] made Einstein to agree that the evolution of the universe is described by the non-stationary Friedmann solution [3] governed by the energy density of the usual matter. After that and till almost the end of the XX century vacuum energy was rejected by the "establishment", though there were a few bright names such as Le Maittre, Eddington, Bronshtein who believed in Lambda. A short renaissance of Lambda took place in the beginning of 60 s when an attempt was done to explain an accumulation of quasars near red-shift $z \approx 2$ by non-zero vacuum energy [4]. Later it was understood that the red-shift distribution of quasars can be well explained with $\rho_{v a c}=0$ and the general attitude to non-zero vacuum energy became even worse than it was before.

Renewed interest to vacuum energy came from quantum field theory. Probably the first published paper on the subject was by Zeldovich [5]. He stressed that the energy of vacuum quantum fluctuations results in infinitely large vacuum energy and suggested that the problem could be solved by cancellation of bosonic and fermionic contributions. This would be indeed true if the world were strictly supersymmetric but, as we know, this is not the case. Supersymmetry, if it was ever realised in nature, would have been broken at the scale $M_{S U S Y} \geq 1 \mathrm{TeV}$ and vacuum energy 
in broken state should be about

$$
\rho_{\text {vac }}^{(S U S Y)} \sim M_{S U S Y}^{4} \geq 10^{12} \mathrm{GeV}^{4}
$$

In the case of locally realised supersymmetry, i.e. supergravity (SUGRA), vacuum energy is allowed to be small but at the expense of unbelievably accurate fine-tuning. The natural value of vacuum energy in such theories is about $\rho_{v a c}^{(S U G R A)} \sim M_{P l}^{4} \sim 10^{76} \mathrm{GeV}^{4}$. To fit the observational data (see below) the fine tuning must be precise with the accuracy of $10^{-123}$ !

A very strong argument in favour of non-trivial vacuum properties is presented by Quantum Chromodynamics (QCD). According to this theory, which perfectly agrees with experiment, the vacuum is not empty. It is filled by quark [6] and gluon condensates [7]. The energy density of these QCD condensates is negative and is about

$$
\left|\rho_{\text {vac }}^{(Q C D)}\right| \sim \Lambda_{Q C D}^{4} \sim 10^{-3} \mathrm{GeV}^{4}
$$

Existence of such condensates is an experimentally established fact and the greatest mystery is what else "lives" in vacuum whose energy compensates $\rho_{v a c}^{(Q C D)}$ down to $10^{-44}$ of the QCD contribution. It cannot be any field related to QCD because all light fields are observed in experiment, while heavy ones simply cannot achieve compensation with such a precision.

So such compensating field, $\Phi$, cannot have colour interaction and most naturally it is coupled to vacuum energy-momentum tensor, $T_{\mu \nu}^{(v a c)}$, through gravity as any field should. But the coupling must be arranged in such a way that the non-zero curvature induced by vacuum creates a condensate of $\Phi$ whose energy in turn kills the source. This is the idea of adjustment mechanism suggested a quarter of century ago [8]. Since then a few dozens of different models of adjustment have been studied, for a review see e.g. ref. [9]. One may also refer to [10] for a broader review on different alternative mechanisms available in the literature. Unfortunately none of the models led to realistic cosmology without an additional fine-tuning. On the other hand, such models successfully compensated a preexisting vacuum energy down to the terms of the order of the critical energy density $\rho_{c} \sim M_{P l}^{2} / t^{2}$. This is a general feature of adjustment mechanism. In this sense the prediction about cosmological dark energy was done long before its discovery by astronomers.

Observational data in favour of non-zero vacuum or, generally speaking, dark energy were accumulated during the last 10 years. There are several independent kinds of observations, which are all best explained if the cosmological energy is dominated by an unknown form of the so called dark energy, which may be simply vacuum energy or something similar to it. One can distinguish between the two by the equation of state expressing the pressure density, $p$, through the energy 
density, $\rho$ :

$$
p=w \rho .
$$

For vacuum energy $w=-1$ and $\rho$ remains constant in the course of cosmological expansion. If $w \neq-1$ and, moreover, $w=w(t)$, such unknown cosmological energy is called dark energy. According to the data $w$ is compatible with $(-1)$ with better than $10 \%$ accuracy [1]|. For an up-to-date and critical review of the current status of observations on the subject see [12].

Here we propose a new model of dynamical adjustment of vacuum energy down to the observed value. The model is based on a scalar field $\Phi$ which is non-minimally coupled to the curvature scalar, $V(\Phi) R$, and in this sense it is close to the original paper [8] but the model avoids many of the shortcomings of that mechanism. In particular, the Weinberg no-go theorem [13] for adjustment models based on a scalar field can be evaded. Moreover, cosmology based on the suggested mechanism may be realistic.

\section{LAGRANGIAN AND EQUATIONS OF MOTION}

The model of adjustment of vacuum energy down to (almost) zero, which is considered here, is based on a simple Lagrangian of a scalar field $\phi$ non-minimally coupled to gravity:

$$
\mathcal{L}=\frac{1}{2}(\partial \phi)^{2}-U(\phi)-V(\phi) R
$$

where $R$ is the curvature scalar.

The equation of motion for field $\phi$ has the form

$$
D^{2} \phi+U^{\prime}(\phi)+R V^{\prime}(\phi)=0
$$

where prime means derivative with respect to $\phi$ and $D$ is the covariant derivative in the gravitational field. The latter is governed by the equation:

$$
\begin{aligned}
2 V(\phi)\left(R_{\mu \nu}-\frac{1}{2} g_{\mu \nu} R\right)= & \partial_{\mu} \phi \partial_{\nu} \phi-\frac{1}{2} g_{\mu \nu}\left[\partial_{\alpha} \phi \partial^{\alpha} \phi-2 U(\phi)\right] \\
& -2\left(g_{\mu \nu} D^{2}-D_{\mu} D_{\nu}\right) V(\phi)+T_{\mu \nu} .
\end{aligned}
$$

where $T_{\mu \nu}$ is the energy-momentum tensor of matter defined by

$$
T_{\mu \nu}=-\frac{2}{\sqrt{g}} \frac{\delta S_{m}}{\delta g^{\mu \nu}}
$$

The Minkowski metric is taken to be $\eta_{\mu \nu}=\operatorname{diag}(1,-1,-1,-1)$, and $g=-\operatorname{det} g_{\mu \nu}$. 
To avoid misundersanding we would like to note that the vacuum energy term $\rho_{v a c} g_{\mu \nu}$ is usually inserted into $T_{\mu \nu}$, but it is evident that it can be added as a constant to $U(\phi)$ as well.

In what follows we will consider a homogeneous case described by the Friedmann-RobertsonWalker (FRW) metric; the field $\phi$ is assumed to be a function of time only.

Taking the trace of eq. (6) and using eq. (5) we find for the curvature scalar:

$$
R=\frac{\dot{\phi}^{2}\left(1+6 V^{\prime \prime}\right)-4 U-6 V^{\prime} U^{\prime}-T_{\mu}^{\mu}}{2\left(V+3 V^{\prime 2}\right)} .
$$

Now the equation of motion for $\phi$ takes the form:

$$
\ddot{\phi}+3 H \dot{\phi}+\frac{\dot{\phi}^{2} V^{\prime}\left(1+6 V^{\prime \prime}\right)+2 U^{\prime} V-4 U V^{\prime}-V^{\prime} T_{\mu}^{\mu}}{2\left(V+3 V^{\prime 2}\right)}=0 .
$$

The Hubble parameter is equal to:

$$
H=-\frac{V^{\prime} \dot{\phi}}{2 V}+\left[\left(\frac{V^{\prime} \dot{\phi}}{2 V}\right)^{2}+\frac{\dot{\phi}^{2}+2 U+2 \rho_{m}}{12 V}\right]^{1 / 2}
$$

where $\rho_{m}$ is the energy density of matter, which we disregard below for a while.

The energy and pressure densities of the system are given, in the perfect fluid approximation, by:

$$
\begin{aligned}
\rho_{\phi} & =\frac{1}{2} \dot{\phi}^{2}+U-6 H \dot{\phi} V^{\prime} \\
P_{\phi} & =\frac{1}{2} \dot{\phi}^{2}\left[1+4 V^{\prime \prime}-\frac{2 V^{\prime 2}\left(1+6 V^{\prime \prime}\right)}{V+3 V^{\prime 2}}\right]-U\left[\frac{V-V^{\prime 2}}{V+3 V^{\prime 2}}\right]+2 H \dot{\phi} V^{\prime}-\frac{2 U^{\prime} V^{\prime} V}{V+3 V^{\prime 2}} .
\end{aligned}
$$

One can see that in the absence of matter or for relativsitic matter, i.e. for $T_{\mu}^{\mu}=0$, the stationary point of equation (9) is a solution of the equation:

$$
U\left(\phi_{s}\right)=\frac{V\left(\phi_{s}\right)}{2 V^{\prime}\left(\phi_{s}\right)} U^{\prime}\left(\phi_{s}\right)
$$

One can easily check that for positive vacuum energy, see below eq. (18), the second derivative of the effective potential in the stationary point $\phi=\phi_{s}$, eq. (13), is positive, i.e. this point is stable. If $T_{\mu}^{\mu} \neq 0$, the position of the stationary point would be shifted according to:

$$
U\left(\phi_{s}\right)+\frac{T_{\mu}^{\mu}}{4}=\frac{V\left(\phi_{s}\right)}{2 V^{\prime}\left(\phi_{s}\right)} U^{\prime}\left(\phi_{s}\right)
$$

Since by construction $T_{\mu}^{\mu}$ includes only normal matter which decrease in the course of cosmological expansion as $1 / a^{3}$, where $a$ is the cosmological scale factor, the omission of the $T_{\mu}^{\mu}$-term does not significantly change the result. 
If by some reason the ratio $V / V^{\prime}$ is small then the value of the effective vacuum energy at equilibrium $U\left(\phi_{s}\right)$ is also small. We will see that this is indeed the case for a certain choice of the potentials $U(\phi)$ and $V(\phi)$ if the original vacuum energy, $\rho_{v a c}$, is large.

There is nothing new in Lagrangian (41). Many models of adjustment starting from ref. [8] used similar $\mathcal{L}$. Moreover, we take the potential $U(\phi)$ in the simplest possible form, namely just the potential of free massive field:

$$
U(\phi)=\rho_{v a c} \pm m_{\phi}^{2} \phi^{2} / 2
$$

Here $\rho_{v a c}$ is the initial vacuum energy density. If $\rho_{v a c}>0$ then we have to choose negative sign in front of the mass term in eq. (15); for negative $\rho_{v a c}$ the sign should be positive. It may seem that negative mass term would lead to instability because the potential $U(\phi)$ is not bounded from below. We will see that it is not so because there is a stable equilibrium point at finite $\phi=\phi_{s}$. As we show in what follows, the resulting vacuum energy at this point, $\rho_{v a c}^{(e f f)}=U\left(\phi_{s}\right)$ is non-vanishing and automatically small, if the initial $\rho_{\text {vac }}$ is large.

The important less trivial input is the form of the coupling of $\phi$ to curvature $R$. We take it in the form:

$$
V(\phi)=m_{P l}^{2} \exp \left(\frac{\phi^{4}-\phi_{s}^{4}}{\mu^{4}}\right)
$$

though other similar types of the potential $V(\phi)$ are possible. Here $m_{P l}^{2}$ is the value of the effective Planck mass at the stationary point of the equations of motion, $\phi=\phi_{s}$. As we shall see shortly, the universe has not yet reached the state where $\phi=\phi_{s}$. Hence the value of the Planck mass now, $M_{P l}$ would be different from that at the stationary point,

$$
M_{P l}^{2}=m_{P l}^{2} \exp \left[\left(\phi_{0}^{4}-\phi_{s}^{4}\right) / \mu^{4}\right]
$$

where $\phi_{0}$ is the value of $\phi$ today.

In what follows we assume that the original vacuum energy is positive,

$$
\rho_{v a c}=M_{v}^{4}>0
$$

and that $M_{v}$ is of the order of $M_{P l}$, while the other relevant mass parameters $m_{\phi}$ and $\mu$ are both of the order of $\mathrm{TeV}$. Such a choice of numerical values is not obligatory but an important thing is that $M_{v} \gg\left(m_{\phi}, \mu\right)$.

The value of $\phi$ at the stationary point is:

$$
\phi_{s}^{2} \approx \frac{2 M_{v}^{4}}{m_{\phi}^{2}}+\frac{\mu^{4} m_{\phi}^{4}}{M_{v}^{4}}-\frac{\mu^{8} m_{\phi}^{6}}{128 M_{v}^{12}} .
$$


The value of the effective vacuum energy in the stationary point would be

$$
U\left(\phi_{s}\right) \approx-\left(\frac{\mu^{2} m_{\phi}^{2}}{4 M_{v}^{2}}\right)^{2} .
$$

With the chosen above "natural" numerical values (Planck scale for the vacuum, TeV scale for the fields) the vacuum energy at the stationary point would be $\left(-10^{-52}\right) \mathrm{GeV}^{4}$, which is not catastrophically far from the observed today density of dark energy, $\rho_{D E} \sim 10^{-47} \mathrm{GeV}^{4}$. More troubling may be the sign difference. However, we will see in the following section that the universe spends cosmologically large time in the state where $U(\phi)$ is positive and close to the observed value (with a reasonable choice of masses $m_{\phi}$ and $\mu$ ). So we have not yet reached the asymptotic stationary point and most probably will never reach it because the cosmological expansion will turn into contraction before $\phi$ reaches $\phi_{s}$.

Let us also stress that such transplanckian value for $\phi_{s}$ is not a problem, since its potential is always smaller that $M_{v}^{4}$, and only powers of $U / M_{v}^{4}$ enter in the effective non-renormalisable potential, see [14]. Notice further that at very early times, when $\phi \ll \phi_{c}$ (see below), this statement is no longer true due to the smallness of $V$, and a more complete quantum gravity description of the system is needed.

\section{SOLUTION OF EQUATIONS OF MOTION}

Numerical solution of eqs. (9) and (10) is difficult because of the huge variation of relevant quantities due to exponential form of $V(\phi)$. Fortunately an approximate analytical solution can be found in the interesting range of $\phi$ values.

One can easily check that when $\phi<\phi_{s}$ and far from it, it rather quickly rises in direction to $\phi_{s}$. During this stage the universe expanded by a huge factor and at this regime $\phi$ plays the role of the inflaton. So inflation is automatically implemented into the model. We can see this by writing down the equation for the acceleration parameter $\ddot{a}$ :

$$
\frac{\ddot{a}}{a}=-H^{2}-\frac{R}{6} \simeq \frac{U-\dot{\phi}^{2}}{6 V},
$$

where the last step refers to the early stage of evolution. When $\phi \ll \phi_{s}$ the acceleration is huge and positive. Inflationary solutions for similar setup were studied in [15], and found to be in excellent agreement with observations. Alternatively, one can make a conformal coordinate transformation $\bar{g}_{\mu \nu}=\left(m_{P l}^{2} / V\right) g_{\mu \nu}$ to the Einstein frame. In such a coordinate system the new gravity lagrangian will be just the usual one $\mathcal{L}_{E}=m_{P l}^{2} \bar{R}$, while the potential for $\phi$ becomes, in close analogy to [15], $\bar{U}=m_{P l}^{4}\left(U / V^{2}\right)$, that is, a hybrid-like potential capable of giving inflationary expansion. 
Moreover, in ref. [15], with a somewhat differ choice for the potentials, a dynamical hierarchy between the effective Planck mass and the electroweak scale was generated. In our case we are primarily interested in a solution to the vacuum energy puzzle, and the variation of the effective Planck mass $m_{P l}^{2}(\phi)=V(\phi)$ at this stage is extremely more drastic. It would be of great interest if it were possible to unify the two proposals, even though at the moment it looks very challenging.

Here we study in some detail the behavior of the solution when $\phi$ approaches $\phi_{s}$, still being sufficiently far from it so that $U(\phi)$ remains positive. We make the expansion

$$
\phi=\phi_{s}+\phi_{1}
$$

where $\phi_{1}<0$ and sufficiently small,

$$
\phi_{1}^{2}<\frac{\mu^{4}}{6 \phi_{s}^{2}} \text { or } \phi_{1}>\phi_{d} \equiv-\frac{\mu^{2}}{\sqrt{6} \phi_{s}} .
$$

If this inequality is fulfilled, we may neglect the terms containing higher powers of $\phi_{1}$ and keep only linear terms. It is convenient to introduce the notation:

$$
V^{\prime}(\phi) / V(\phi)=4 \phi^{3} / \mu^{4} \equiv K(\phi)
$$

and $K \equiv K\left(\phi_{s}\right)$. For a small $\left|\phi_{1}\right|$ the potential $V(\phi)$ can be written as

$$
V(\phi) \approx m_{P l}^{2} \exp \left(K \phi_{1}\right)
$$

while quadratic and higher terms in the exponent can be neglected.

When $\phi$ is sufficiently far from $\phi_{s}$ the following conditions are fulfilled:

$$
V^{\prime \prime} \ll 1 \text {, and }\left(V^{\prime}\right)^{2} \ll V \text {, }
$$

and the equations of motion are significantly simplified. The boundary value of $\phi_{1}=\phi_{c}$, when $V^{\prime \prime}(\phi)=1$ is reached, is equal to:

$$
\phi_{1}<\phi_{c} \equiv-\frac{2}{K} \ln \left(K m_{P l}\right)
$$

Conditions (23) and (27) are compatible if $\left|\phi_{d}\right|>\left|\phi_{c}\right|$. This is true when

$$
m_{P l} K \simeq \frac{m_{P l} M_{v}^{6}}{\mu^{4} m_{\phi}^{3}} \lesssim \exp \frac{M_{v}^{4}}{\mu^{2} m_{\phi}^{2}} .
$$

These conditions simplify the solution of the equations of motion but they are not necessary otherwise and the model may still operate even if they are not fulfilled. 
We shall remark on the following detail before studying the different regimes of interest. Notice that in order to expand further the exponential in (25) as $1+4 \phi_{s}^{3} \phi_{1} / \mu^{4}+\ldots$ we would need $\left|\phi_{1}\right| \ll \mu^{4} / \phi_{s}^{3}$. Since, as it will be soon clear, the interesting cosmological epochs are close to $\left|\phi_{c}\right| \gg \mu^{4} / \phi_{s}^{3}$ we are not allowed to do so. This is to say that the exponential factor plays a key rôle in our setup.

When $\phi_{1}$ approaches $\phi_{c}$ being still larger by the absolute value, i.e. $\left|\phi_{1}\right|>\left|\phi_{c}\right|$, equation (9) takes the form:

$$
\ddot{\phi}_{1}+\frac{\sqrt{3}}{2} \dot{\phi}_{1}\left(\frac{\dot{\phi}_{1}^{2}+2 U}{V}\right)^{1 / 2}+U^{\prime}+\frac{V^{\prime}}{2 V}\left(\dot{\phi}_{1}^{2}-4 U\right)=0 .
$$

Remember that $V \sim \exp \left(K \phi_{1}\right)$ is exponentially small.

Potential (vacuum-like) energy at this stage is positive and is equal to:

$$
U \approx-\frac{\mu^{4} m_{\phi}^{4}}{16 M_{v}^{4}}-m_{\phi}^{2} \phi_{s} \phi_{1} .
$$

In the equation of motion (29) the first constant term in $U$ cancels with the $U^{\prime} V / 2 V^{\prime}$ and only the term proportional to $\phi_{1}$ remains. Moreover, when $\phi_{1}$ approaches to $\phi_{c}$ from below, the first term in $U$, eq.(30), is small in comparison with the second one and at $\phi_{1}=\phi_{c}$ the potential is approximately:

$$
U\left(\phi_{s}+\phi_{c}\right) \approx \frac{\mu^{4} m_{\phi}^{4}}{4 M_{v}^{4}} \ln K m_{P l} .
$$

Such vacuum energy is even closer to the observe value thanks to the large value of $K$ in the logarithm. It can be easily tuned down to the present day cosmological constant e.g. by increasing $m_{\phi}$ by a factor of 3 .

Since the Hubble parameter at this stage is very big, the equation of motion can be solved in slow roll approximation:

$$
\begin{aligned}
\ddot{\phi} & \ll 3 H \dot{\phi}+R V^{\prime}+U^{\prime}, \\
\dot{\phi}^{2} & \ll 2 U .
\end{aligned}
$$

Within this approximation we find

$$
\dot{\phi}_{1}=\sqrt{\frac{8}{3}} m_{P l} m_{\phi} K \sqrt{-\phi_{s} \phi_{1}} e^{K \phi_{1} / 2} .
$$

One should check of course that the neglected terms are sub-dominant, which is indeed the case.

Introducing new dimensionless function $z=K \phi_{1}$ and frequency:

$$
\omega_{1}=\frac{16 \sqrt{2}}{\sqrt{3}} \frac{m_{\phi} m_{P l} \phi_{s}^{5}}{\mu^{6}}
$$


we arrive at a very simple equation:

$$
\dot{z}=\omega_{1} \sqrt{-z} e^{z / 2}
$$

The characteristic time of evolution defined as $\tau_{1}=1 / \omega_{1}$ is small even in microscopic scale to say nothing of the cosmological one. Though one should remember about exponentially small factor, $\exp (z / 2)$, which makes effective time much larger, it still seems non trivial to make it longer then the age of the Universe. In fact, for large $z$, the evolution is indeed slow enough, but at the same time the effective Planck mass becomes extremely tiny, thereby rendering our description unreliable. Nevertheless it might still be possible to find a solution which looks like our universe in the close proximity of $\phi_{c}$. We comment on this possibility in the concluding section.

Let us now turn our attention to equation (9) at the stage when $V^{\prime \prime} \gg 1$ and $\left(V^{\prime}\right)^{2} \gg V$, when the equation takes the form:

$$
\ddot{\phi}+3 H \dot{\phi}+\frac{V^{\prime \prime}}{V^{\prime}} \dot{\phi}^{2}+\frac{U^{\prime} V-2 U V^{\prime}}{3\left(V^{\prime}\right)^{2}}=0 .
$$

One can check that the term $\dot{\phi}^{2} V^{\prime \prime} / V^{\prime}$ enforces very small value of $\dot{\phi}$ and thus the Hubble parameter takes approximately the standard form, proportional to square root of the energy density. One should of course verify a posteriori that these terms can be neglected. For $\left|\phi_{1}\right|<\left|\phi_{c}\right|$ the error we make is of order $20 \%$.

Neglecting $\ddot{\phi}$ and $\left(\dot{\phi} V^{\prime} / 2 V\right)^{2}$ in $H$ we obtain the following equation:

$$
\dot{\phi}=\frac{\sqrt{6}}{9} \frac{V^{3 / 2}}{\left(V^{\prime}\right)^{2}} \frac{2 K U-U^{\prime}}{\sqrt{U}} .
$$

Now both terms in $U$ are essential, see eq. (30), while the factor $\left(2 K U-U^{\prime}\right)$ takes the form:

$$
2 K U-U^{\prime} \approx-2 K m_{\phi}^{2} \phi_{s} \phi_{1}\left(1-\frac{1}{2 K \phi_{1}}\right) \approx-2 K m_{\phi}^{2} \phi_{s} \phi_{1} .
$$

Our approximation breaks down when $U \simeq 0$, or, better to say, when $2 K \phi_{1} \simeq 1$, i.e. when

$$
\phi_{1}^{(0)}=-\frac{\mu^{4} m_{\phi}^{3}}{16 \sqrt{2} M_{v}^{6}} .
$$

When $\phi_{1}$ approaches $\phi_{1}^{(0)}$, the $\dot{\phi}^{2}$-terms in $H$ should be taken into account. The potential energy at $\phi_{1}^{(0)}$ is still positive but extremely tiny

$$
U_{0}=\frac{\mu^{8} m_{\phi}^{8}}{1024 M_{v}^{12}} .
$$

At some moment, very soon after $\phi_{1}$ has crossed $\phi_{1}^{(0)}$, the total energy density will vanish, $H$ will become zero, and the cosmological expansion will stop and turn into contraction. Our universe 
has not yet reached this stage and we will take $\phi_{1}$ far from $\phi_{1}^{(0)}$ and neglect the constant term in the potential (30).

Introducing again $z=K \phi_{1}$ we find the equation very similar to eq. (34):

$$
\dot{z}=\omega_{2} \sqrt{-z} e^{-z / 2}
$$

Here

$$
\omega_{2}=\frac{1}{3 \sqrt{3}} \frac{m_{\phi}^{2} \mu^{2}}{m_{P l} M_{V}^{2}} .
$$

Notice that the sign of the exponent is opposite to that in eq. (34).

The characteristic time of evolution

$$
\tau_{2}=\frac{1}{\omega_{2}}=10^{21} \mathrm{~s} \frac{m_{\phi}^{2} \mu^{2}}{\mathrm{TeV}^{4}} \frac{\left(10^{16} \mathrm{TeV}\right)^{3}}{m_{P l} M_{v}^{2}},
$$

which is rather close to the cosmological time. The evolution of $z$ is much faster for very large negative $z$ but if $z \sim(-10)$ we remain with long effective time of variation and still far from $\phi_{1}^{(0)}$, since $z^{(0)}=K \phi_{1}^{(0)}=-1 / 2$.

The time variation of the Newtonian constant at this epoch is

$$
\frac{\dot{G}_{N}}{G_{N}}=-\dot{z}
$$

Using $m_{\phi}=3 \mathrm{TeV}$, according to eq. (40) it is smaller than $10^{-19} / \mathrm{sec}$ for $z=-10$ and is perfectly below the existing bounds [16]. See also the recent paper [17] for more stringent, although speculative, limits.

It is noteworthy that at the stage when $\phi_{1}$ is still far from $\phi_{1}^{(0)}$, the vacuum-like energy is positive, noticeably larger than that given by eq. (39), and rather naturally close to the observed value.

The problem with this second evolutionary regime is that, despite the presence of vacuum energy of the correct magnitude, the Universe is not accelerating. It is easy to see how this comes about by looking again at equation (21), which in the regime of interest here reads $\ddot{a} / a \simeq-U / 6 \mathrm{~V}$, thereby implying a negative acceleration. One can also check that this must be the case by the expressions for energy and pressure densities (11) and (12), which in this regime can be cast in the form

$$
\begin{aligned}
\rho_{\phi} & \simeq U-6 H \dot{\phi} V^{\prime}, \\
3 P_{\phi} & \simeq U+6 H \dot{\phi} V^{\prime} .
\end{aligned}
$$

Using (36) (and accounting for the error made in that approximation), the equation of state therewith obtained confirms this result. 


\section{DISCUSSION/CONCLUSION}

We proposed here a model of dynamical adjustment of vacuum energy which quite successfully achieves this goal, however at the high price of a too fast time variation of the gravitational coupling strength. Vacuum energy is compensated from initially huge value down to some very small non-zero residual vacuum energy which magnitude is naturally of the order of that observed today. The smallness of remaining $\rho_{v a c}$ is dictated by its huge initial value - the surviving remnant of $\rho_{v a c}$ is inversely proportional to its initial magnitude. Of course there remain the problem of coincidence of vacuum energy today and the energy density of the normal matter, which has not been considered here.

The asymptotic value of vacuum energy is constant and negative and it may seem to be in contradiction with the data. However, the evolution of the compensating field, which was very fast initially, drastically slows down at later cosmological stage so that the universe remains with effective and slowly time-changing effective vacuum energy during very long time which can be easily larger than the universe age $t_{U} \approx 5 \cdot 10^{17}$ sec. However in such a regime the Universe would be decelerating, in contrast to what we observe.

Going further, the model predicts that in a very distant future the universe will approach negative total energy and the cosmological expansion will turn into contraction. This unexpected feature differentiates our model from other adjustment scenarios but, unfortunately there is not enough time in at our disposal to observe such a contraction.

For positive initial vacuum energy the potential of field $\phi$ is not bounded from below, $U(\phi)=$ $-m_{\phi}^{2} \phi^{2}+\rho_{v a c}$ but this feature does not lead to any undesirable consequences because the equation of motion has a stable minimum at finite $\phi=\phi_{s}$ where the energy density is finite as well.

We have not considered realistic cosmology with the usual matter. Cosmology with relativistic matter does not appear too tricky a problem, because $T_{\mu}^{\mu}=0$ and does not enter into expression for curvature (8). In the regime when $\left(V^{\prime}\right)^{2} \gg V$, we obtain $R \ll H^{2}$, which is true for the usual relativistic expansion, when $R=0$ and $H=1 / 2 t$. However, if we have nonrelativistic matter at the epoch when $\left(V^{\prime}\right)^{2} \gg V$, then again $R \ll H^{2}$ and the regime of cosmological expansion would be determined again by $H \approx 1 / 2 t$ which differs from the usual one with $H=2 / 3 t$.

However at the moment a more urgent and serious problem is the too fast evolution of the effective Planck mass in the first regime. Possibly for realistic cosmology we need to study some other forms of potentials $U$ and $V$ which would allow to have a slow expansion regime when $\left(V^{\prime}\right)^{2} \ll$ $V$. On the other hand, this may be achieved by equipping the field with some decay channels or 
by studying the effects (enhancement of friction term) of gravitational particle production in that regime. If this could indeed be engineered, also matter would be readily and easily introduced.

Acknowledgments We wish to thank C. Bambi for collaborating on the early stage of this work.

[1] A. Einstein, Sitzgsber. Preuss. Acad. Wiss. 1 (1918) 142.

[2] E.P. Hubble, Proc. Nat. Acad. Sci. 15 (1929) 168.

[3] A. Friedman, Z. Phys. 10 (1922), 377.

[4] V. Petrosian, E.E. Salpeter, P. Szekeres, Astrophys. J. 147 (1967) 1222;

I. Shklovsky, Astrophys. J. 150 (1967) L1;

M. Rowan-Robinson, MNRAS, 141 (1968) 445.

[5] Ya.B. Zeldovich, Uspekhi Fiz. Nauk, 95 (1968) 209.

[6] M. Gell Mann, R.J. Oakes, B. Renner, Phys. Rev. 175 (1968) 2195.

[7] M.A. Shifman, A.I. Vainshtein, V.I. Zakharov, Nucl. Phys. B147 (1978) 385.

[8] A.D. Dolgov, in The Very Early Universe, ed. G.Gibbons, S.W.Hawking, and S.T.Tiklos (Cambridge University Press, 1982).

[9] A. D. Dolgov, arXiv:hep-ph/0405089, Published in "La Thuile 2004, Results and perspectives in particle physics", 75-94;

A. D. Dolgov, arXiv:hep-ph/0203245.

[10] E. J. Copeland, M. Sami and S. Tsujikawa, Int. J. Mod. Phys. D 15, 1753 (2006) arXiv:hep-th/0603057.

[11] D. N. Spergel et al. [WMAP Collaboration], Astrophys. J. Suppl. 170, 377 (2007) arXiv:astro-ph/0603449].

[12] S. Sarkar, arXiv:0710.5307 [astro-ph].

[13] S. Weinberg, Rev. Mod. Phys. 61, 1 (1989).

[14] A. D. Linde, arXiv:hep-th/0503203.

[15] T. Biswas and A. Notari, Phys. Rev. D 74 (2006) 043508 arXiv:hep-ph/0511207;

F. Di Marco and A. Notari, Phys. Rev. D 73 (2006) 063514 arXiv:astro-ph/0511396.

[16] J. G. Williams, S. G. Turyshev and D. H. Boggs, Phys. Rev. Lett. 93, 261101 (2004) arXiv:gr-qc/0411113;

T. Clifton, D. F. Mota and J. D. Barrow, Mon. Not. Roy. Astron. Soc. 358, 601 (2005) arXiv:gr-qc/0406001.

[17] C. Bambi and A. Drago, arXiv:0711.3569 [hep-ph]. 